\title{
Ultra-Wide Range Wavelength Conversion Using FWM in a Raman DFB Fiber Laser
}

\author{
Jindan Shi, Shaif-ul Alam, and Morten Ibsen \\ Optoelectronics Research Centre, University of Southampton, Highfield, Southampton, SO17 1BJ, UK \\ jxs@orc.soton.ac.uk
}

\begin{abstract}
We demonstrate for the first time to our knowledge, four-wave-mixing (FWM) in a $30 \mathrm{~cm}$-long center $\pi$ phase-shifted Raman distributed-feedback (DFB) fiber laser. The FWMto-signal conversion efficiency is $-24 \mathrm{~dB}$ and the wavelength conversion range is $94.1 \mathrm{~nm}$.

(C) 2012 Optical Society of America

OCIS codes: (060.3735) Fiber Bragg gratings; (190.4380) Nonlinear optics, four-wave mixing;
\end{abstract}

\section{Introduction:}

Four-wave mixing (FWM) is an attractive approach to generate radiation at new frequencies through the interaction between light at two or three different incident frequencies, with the features of (i) the converted signal is identical to the original; (ii) the tuning range is continuous; (iii) transparent in terms of modulation format; (iv) simultaneous conversion of multiple signals [1-3]. There are several mediums which can be used for efficient FWM process, however optical fiber is unique in the sense that it offers long interaction length thereby reducing the demand on optical intensity. Additionally, fibers offers low insertion loss when integrated into optical transmission systems. Typically, a strong pump signal and a weak probe signal are injected into a long length (more than a few meters) of dispersion tailored fiber and a new signal called idler or conjugate wave is generated through the FWM process. A more compact method to obtain FWM would be to use a DFB fiber laser as a mixing device and use the signal of the DFB laser as the pump for the FWM process. Such mixing process has previously been reported in the context of semiconductor DFB lasers [3, 4]. However, so far, no FWM-related wavelength conversion has been observed in DFB fiber lasers. Recently, Raman DFB (R-DFB) fiber lasers with watt-level threshold and high output power have been experimentally demonstrated [5]. In that particular design, the phase-shifted DFB grating forms a high reflectivity resonating cavity for the R-DFB signal, whilst being mostly transparent to the pump wave. In addition, the dispersion at the DFB signal wavelength is highly tailored by the DFB grating structure [6]. In this work, we report, for the first time, the observation of efficient partially degenerated FWM with an ultra-wide detuning range in a 30cm-long Raman DFB fiber laser.

\section{Experimental setup}
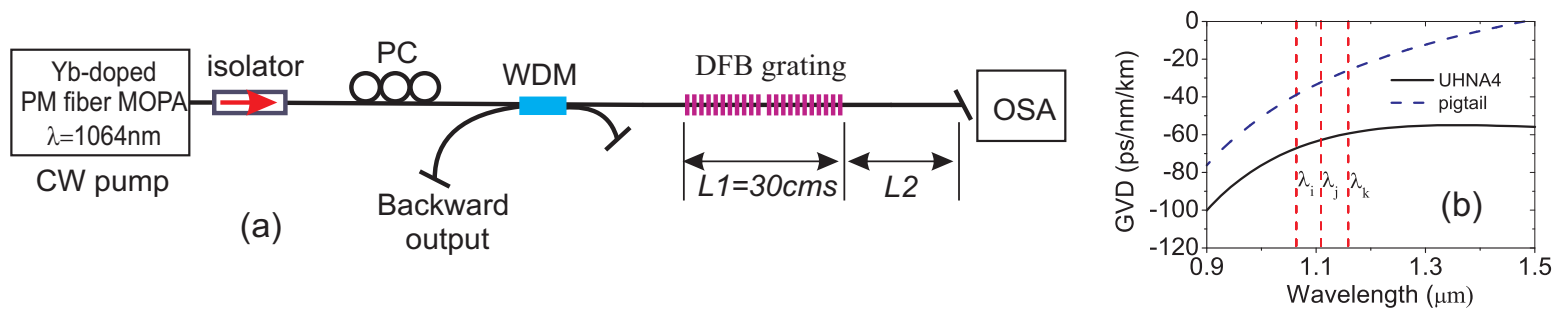

Fig. 1 (a) Schematic diagram of experimental setup; (b) Calculated total group-velocity-dispersion (GVD) of fibers.

The schematic of the experimental setup is shown in Fig. 1(a). A 1064.6nm continuous-wave (CW), linearly polarized, Yb-doped PM fiber MOPA was used as the pump source of the R-DFB laser. The output was spliced to a non-PM high power isolator whilst a polarization controller (PC) was used to align the pump polarization state to the DFB grating. A $1064 \mathrm{~nm} / 1117 \mathrm{~nm}$ wavelength division multiplexer (WDM) was used to monitor the backward R-DFB signal from the system. The forward output of the DFB grating was spliced to a single-mode fiber (PS980 from Fibercore Ltd.) with a length of L2 which was directly coupled into an optical spectrum analyzer (OSA). All the fiber ends were angle-cleaved to prevent end-feedback while the WDM and DFB grating were mounted on heat sinks to help control the temperature and better remove any generated heat.

We used the same R-DFB grating sample $(30 \mathrm{cms}$ long with center $\pi$ phase shift formed in Nufern UHNA4 fiber) as used in our previous work [7]. The NA, propagation loss $(\alpha)$ at $1.1 \mu \mathrm{m}$ and nonlinear coefficient $(\gamma @$ $1.1 \mu \mathrm{m})$ are estimated to be $0.35,5 \mathrm{~dB} / \mathrm{km}$ and $\sim 34(\mathrm{Wkm})^{-1}$, respectively.

\section{Results and discussion:}

Fig. 1(b) shows the calculated total group-velocity-dispersion (GVD) of the DFB grating fiber (UHNA4) and the pigtail (L1 \& L2). The three vertical dashed lines respectively, indicate the wavelengths of the pump-source wavelength for the R-DFB fiber laser $\left(\lambda_{i}=1064.6 \mathrm{~nm}\right)$, the R-DFB oscillating wavelength $\left(\lambda_{j}=1109.7 \mathrm{~nm}\right)$ determined by the DFB grating, and the wavelength of the converted signal $\left(\lambda_{k}=1158.7 \mathrm{~nm}\right)$, according to the 
relationship of $\frac{c}{\lambda_{k}}=2 \frac{c}{\lambda_{j}}-\frac{c}{\lambda_{i}}$, where $\mathrm{c}$ is the speed of light in vacuum. As seen in Fig. 1(b), all the wavelengths are located within the normal dispersion region of the used fibers. Fig. 2(a) shows the forward and backward output spectra with the resolution bandwidth (RBW) of $1 \mathrm{~nm}$ with an $L 2$ of $1.8 \mathrm{~m}$. The inserts in Fig. 2(a) are measured with a RBW of $0.01 \mathrm{~nm}$. Note that a free-space attenuator (wavelength independent) was applied before launching the forward output into the OSA. From the forward output spectrum, three waves at wavelengths of $\lambda_{i}, \lambda_{j}$ and $\lambda_{k}$ are clearly seen with $>40 \mathrm{~dB}$ signal-to-noise ratio (SNR) and narrow $3 \mathrm{~dB}$ bandwidths of $<0.1 \mathrm{~nm}$ ascertained from the insets in Fig. 2(a). The Stokes wave at $1158.7 \mathrm{~nm}\left(\lambda_{k}\right)$ is the FWM signal generated from the interaction of the pump laser for R-DFB laser $\left(\lambda_{i}\right)$ and the forward R-DFB signal $\left(\lambda_{j}\right)$ rather than the second order Raman Stokes laser since no cavity exists for this wavelength. Additionally it does not appear at the backward output indicating that it is not a lasing signal from the R-DFB. In order to investigate that the FWM signal is generated entirely within the R-DFB fiber laser and not in, or partially in, the pigtail ( $L 2$ in Fig. 1(a)), we also measured the output spectra with a length of $L 2$ of $11.8 \mathrm{~m}$, and the output spectra were found to be identical to the case of $L 2=1.8 \mathrm{~m}$. Consequently, we conclude that the FWM is generated entirely inside the R-DFB fiber laser cavity. As also evident from the insets of Fig. 2(a), the FWM signal is converted from the pump wave, indicating that the R-DFB signal in this case acts as a pump for the FWM process. The detuning range from the pump signal to the FWM generated signal is $47 \mathrm{~nm}$ corresponding to the frequency detuning of 11.4THz. Fig. 2(b) shows the power ratio $(r)$ between the FWM generated signal $\left(\lambda_{k}\right)$ to its original wave $\left(\lambda_{i}\right)$ for increasing with the incident pump power, measured from the OSA traces with RBW of $1 \mathrm{~nm}$, When the incident pump power exceeds $\sim 2 \mathrm{~W}, r$ levels off at around $-24 \mathrm{~dB}$. This conversion efficiency is as high as that obtained at a wavelength detuning of just $3.8 \mathrm{~nm}$ in a single-mode fiber of $10 \mathrm{~km}$ length pumped at its zero-dispersion wavelength [2]. In the case of the R-DFB the high conversion efficiency and wide detuning range is believed to be due to the resonance effect of the DFB cavity combined with the tailored dispersion properties of the fibre at the R-DFB wavelength induced by the $\pi$ phase-shifted grating [6].
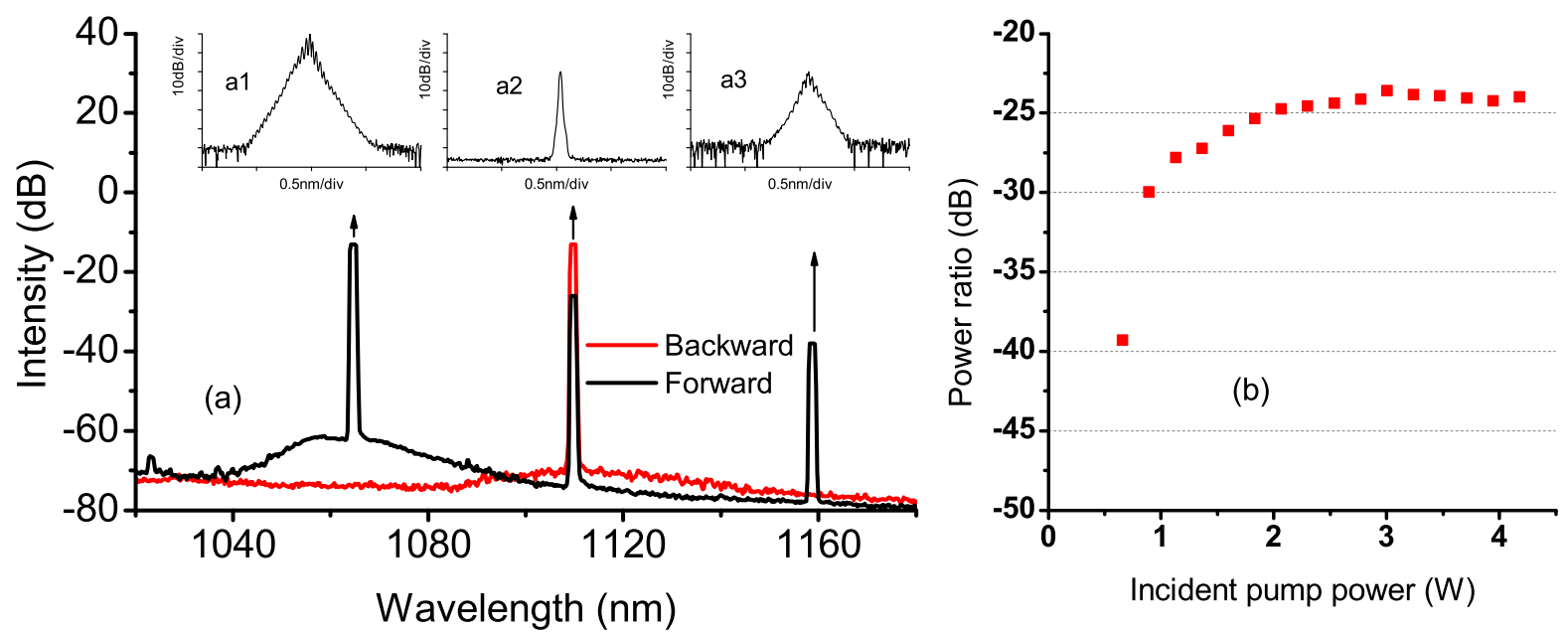

Fig. 2 (a) Forward and backward output spectra at anincident pump power of $4.2 \mathrm{~W}$ with RBW of 1 nm. Insets above are the spectra of the pump (a1), R-DFB (a2) and FWM (a3) waves with RBW of $0.01 \mathrm{~nm}$, respectively; (b) Power ratio with respect to incident pump power.

\section{Conclusion}

In summary, we have demonstrated highly efficient FWM-based wavelength conversion in a 30cm long Raman DFB fiber laser. The conversion efficiency is found to be $-24 \mathrm{~dB}$ for a detuning frequency of $11.4 \mathrm{THz}$.

\section{References:}

1. G. P. Agrawal, "Nonlinear fiber optics," 2nd Edition (Academic Press) (1995).

2. K. Inoue and H. Toba, "Wavelength conversion experiment using fiber four-wave mixing," Photonics Technology Letters, 4, pp. 69-72, (1992).

3. J. Minch, C. S. Chang, and S. L. Chuang, "Four-wave mixing in a distributed-feedback laser," Applied Physics Letters 70, pp. 1360-1362, (1997).

4. H. Kuwatsuka, H. Shoji, M. Matsuda, and H. Ishikawa, "Nondegenerate four-wave mixing in a long-cavity $\lambda / 4$-shifted DFB laser using its lasing beam as pump beams," Journal of Quantum Electronics, 33, pp. 2002-2010 (1997).

5. J. Shi, S.-u. Alam, and M. Ibsen, "High power, low threshold, Raman DFB fibre lasers," in proceedings to IQEC/CLEO Pacific Rim Sydney, Australia 28 Aug - 1 Sep 2011, postdeadline paper, (2011).

6. Z. Qingsheng, "Dispersive properties in phase-shifted Bragg grating filters," in Antennas and Propagation Society International Symposium 1998 IEEE, 1062, pp. 1060-1063, (1998).

7. J. Shi, S. -u Alam, and M. Ibsen, "Highly Efficient Raman Distributed Feedback Fibre Lasers," Accepted for publication in Optics Express (2012). 